\title{
Investigations of characteristics of metal film based pyroelectric detectors implanted by Ar+ Ions
}

\author{
V.O. Lysyuk, L.V. Poperenko, V.S. Staschuk \\ Taras Shevchenko Kyiv National University, 6 Glushkova prosp., 03127 Kyiv, Ukraine, \\ Phone: +38(044) 266 2296; fax +38(044)266 4507; e-mail: lysiuk@mail.univ.kiev.ua, plv@phys.univ.kiev.ua
}

\begin{abstract}
Functional parameters and characteristics of pyroelectric detectors based on the system thin metal film - thick pyroelectric substrate are investigated. Such metals as Ni, Mo and Ti were used to increase absorption of such systems. The pyroelectric substrates $\left(\mathrm{LiNbO}_{3}\right.$ and $\mathrm{LiTaO}_{3}$ ) with thickness of $0.1 \mathrm{~mm}$ coated by metal films with thicknesses of $15-40 \mathrm{~nm}$ were investigated. Influence of ion implantation on functional characteristics (radiation stability, adhesion) of pyroelectric detectors developed on the base of such systems are shown. Application of such pyroelectric detectors to registration of power laser radiation is proposed.
\end{abstract}

Keywords: pyroelectric detector, ion implantation, thin metal film, spectral sensitivity.

Paper received 14.03.02; revised manuscript received 03.12.02; accepted for publication 17.12.02.

\section{Introduction}

The development of measuring devices and necessity of its application in the automated opto-electronic devices places new stringent requirements upon the characteristics of these devices [1,2]. As a result of vigorous development of laser technologies the humanity has become interested in manufacturing detectors with high radiation stability and fast response. However, the present detectors does not satisfy such requirements [3]. The increase of radiation stability and decrease of time response occured at the expense of decreasing sensitivity of detectors. Naturally, that is not appropriate for high-precision measurements. The pyroelectric detectors meet such requirements the most satisfactorily. Only they can register very short pulses (up to $1 \mathrm{~ns}$ ) without any noticeable distortion. To increase the sensitivity during manufacturing pyroelectric detectors, an absorptive cover is put on the surface of a pyroelectric crystal [4]. The influence of stuffs of the pyroelectric crystal and the absorptive cover as well as manufacture technology of detectors on their functional characteristics are reviewered here. A way to increase radiation stability without noticeable deterioration of other detector characteristics is also described.

\section{Procedure of the sensitive elements production for pyroelectric detectors}

The substrates with a thickness of $100 \mu \mathrm{m}$ were prepared from the $\mathrm{LiNbO}_{3}$ single crystal. Nickel, molibdenum and titanium films with thicknesses of 15,20,30 and $40 \mathrm{~nm}$ were deposited on this substrate by cathode sputtering method. At the lowest film thicknesses the island-like surface structure of such systems was observed. At the film thickness of $60 \mathrm{~nm}$ and greater a sharp absorption of systems thin metal film - thick lithium niobate substrate for appropriate films was registered, and, on the whole, the sensitivity of such film - substrate systems was decreased. The requirements on the minimum chemical activity and sufficient absorption in the IR were included in the criterion for a selection of film materials. Ion implantation of the systems: thin $\mathrm{Ni}$ film - thick $\mathrm{LiNbO}_{3}$ substrate, thin Mo film - thick $\mathrm{LiNbO}_{3}$ substrate and thin Ti film - thick $\mathrm{LiNbO}_{3}$ substrate was carried out in a Vesuviy 2/450 installation by $\mathrm{Ar}^{+}$ions with the energies of $100 \mathrm{keV}$ and the doses of $10^{16} \mathrm{~cm}^{-2}$.

It was necessary to reach a maximum adhesion of the film to the substrate without essential changes in the structure and deterioration of the properties of absorbing film 


\section{V.O. Lysyuk et al.: Investigations of characteristics of metal film...}

and $\mathrm{LiNbO}_{3}$ substrate as well. Theoretical calculations of a profiles of distribution of recoil atoms at the implantation by $\mathrm{Ar}^{+}$ions with energies of 50,100 and $150 \mathrm{keV}$ were performed by the Monte-Carlo method. It is shown that at the energy of $\mathrm{Ar}^{+}$ions of $50 \mathrm{keV}$ the peak of such distribution function lies at a depth of $25 \mathrm{~nm}$; at an energy of $100 \mathrm{keV}$ at a depth of $40 \mathrm{~nm}$; and at an energy of $150 \mathrm{keV}$ at a depth of $80 \mathrm{~nm}$. It is necessary to note that the profiles calculated for $\mathrm{Ni}$, Mo and Ti film on thick $\mathrm{LiNbO}_{3}$ substrate are slightly differed. This result may correspond to weak differences in densities of such materials.

It is also necessary to note that at ion energies of $E>400 \mathrm{keV}$ sharp changes in the structure of samples with deterioration of their optical properties were observed. At sufficiently small ion energies $E<25 \mathrm{keV}$ the implantation practically doesn't essentially influence the surface structure of the samples. The doses of $\mathrm{Ar}^{+}$ions were from $10^{15}$ to $10^{17} \mathrm{~cm}^{-2}$.

Sufficiently larger doses caused the changes in the structure and the properties of the samples and the deterioration of their functional characteristics. Long annealing was carried out at the temperature of $500 \mathrm{~K}$ to decrease the inhomogeneities that appeared in the deposition of the metal films on the substrate and then during further ion implantation.

\section{Estimation of the parameters of pyroelectric detectors}

The principle of the functioning of a pyroelectric detector is based on the pyroelectric effect that consist in the change of the polarizability $P$ of a pyroelectric crystal when varying of its temperature. The current density that appears on a change of temperature $T$ during time $t$ is determined by the expression

$j=\gamma \frac{d T}{d t}$

where $\gamma=\frac{d P}{d t}$ is the pyroelectric coefficient. The large value of pyroelectric coefficient $\gamma$ of a pyroelectric crystal causes sufficiently high sensitivity of a pyroelectric detector. It is well known that such ferroelectrics as lithium niobate $\left(\mathrm{LiNbO}_{3}\right)$ and lithium tantalate $\left(\mathrm{LiTaO}_{3}\right)$ are related to these crystals. The minimum thickness of the sensitive element of ferroelectric crystals has to be equal to $5 \mu \mathrm{m}$. At less film thicknesses, ferroelectric transforms into a linear pyroelectric and the coefficient $\gamma$ is sharply decreases.

The current density is determined by a value of average deviation of the temperature of the sensitive element and by a velocity of a change of the temperature. With respect to the frequency of the IR radiation modulation the average deviation of the temperature is directly proportional, while the velocity of its change is inversely proportional. The action of these two factors is compen- sated within a wide frequency range. The small lag of a pyroelectric detector in comparison with other detectors is just explaned by this compensation. The time response $\tau$ of a pyroelectric detector is determined by the ratio

$$
\tau=\frac{\sqrt{3}}{2 \pi f_{0.5}}
$$

where $f_{0.5}$ is the frequency at which the amplitude of output signal is decreased twice.

$f_{0.5}=\frac{c K}{\pi C_{0}^{2}}$

where $c$ is the heat capacity of a pyroelectric crystal, $K$ is the coefficient of heat transport, and $C_{0}$ is the heat capacity of the absorptive coating. The lag of the pyroelectric detectors is independent of a thickness of the sensitive element in accordance with relationships (2) and (3).

The spectral sensitivity is a very important characteristic of a detector. For pyroelectric detectors it is determined by spectral reflectance of an absorptive layer and design of the detector (cavernous pyroelectric detector of type of a wedge, cone, orb etc.). The pyroelectric detectors can be made with more equable zonal sensitivity than bolometers or termocouples. Such pyroelectric detectors are characterized by high threshold sensitivity, large value of a conversion coefficient, broad dynamic range, capability of unlimited variation of the design forms and functional connections.

Let's write a known formula for determination of a volt-watt sensitivity $S$ of a pyroelectric detector

$$
S=\frac{A_{0} \omega \varepsilon_{1} \gamma \tau_{e}}{\sqrt{C_{0} G\left(1+\omega^{2} \tau_{T}^{2}\right)\left(1+\omega \tau_{e}\right)}}
$$

where $A_{0}$ is the area of pyroelectric detector, $\varepsilon_{1}$ the absorbance of an electrode, $\tau_{e}=C_{0} R_{0}$ the electric time constant, $C_{0}$ the capacity of the shunted crystal and input circuit, $R_{0}$ the resistance shunted of a crystal $R_{c r}$ and load $R_{1}, \tau_{T}=C / G$ the thermal time constant, $C$ the thermal capacity of a crystal, $G$ the factor of thermal losses.

Let's estimate the values of the time constants $\tau_{T}$ and $\tau_{e}$ for sensing devices on the basis of the lithium niobate crystal. In that case, when the factor of thermal losses is determined only by radiation $G=4 \varepsilon_{1} \sigma_{c} T_{0}^{3} A_{0}\left(\sigma_{c}\right.$ is the Stefan-Boltzmann constant, $T_{0}$ is the temperature), and $R_{1}<<R_{c r}$ it is possible to write:

$$
\begin{aligned}
& \tau_{T}=\frac{C_{1} d}{4\left(\varepsilon_{1}+\varepsilon_{2}\right) T_{0}^{3} \sigma_{c}} \\
& \tau_{e}=\frac{\varepsilon \varepsilon_{0} A_{0} R_{l}}{d}
\end{aligned}
$$

where $\mathrm{d}$ is the thickness of a crystal, $C_{1}$ the specific thermal capacity of a crystal, $\varepsilon_{2}$ the radiation emittance of an electrode, $\varepsilon$ the inductivity of a crystal. 
If we take into account that $A_{0}=8 \cdot 10^{-6} \mathrm{~m}^{2}, d=$ $=2.5 \cdot 10^{-5} \mathrm{~m}, \varepsilon_{1}+\varepsilon_{2}=1, \varepsilon=84, R_{1}=10^{9} \mathrm{Ohm}, C_{1}=$ $=3.2 \cdot 10^{6} \mathrm{~J} /\left(\mathrm{m}^{3} \mathrm{~K}\right), \sigma_{c}=5.67 \cdot 10^{-8} \mathrm{~J} /\left(\mathrm{m}^{2} \mathrm{sK}^{4}\right)$, then we shall obtain $\tau_{T}=13 \mathrm{~s}, \tau_{e}=0.23 \mathrm{~s}$.

Let's assume that noises of pyroelectric detector are mostly thermal or Johnson's, then the expression for threshold sensitivity $P$ becomes

$P=\sqrt{\bar{W}_{T}^{2}+\frac{\bar{\alpha}^{2}}{S^{2}}}$

where $W_{T}^{2}=4 k T_{0}^{2} A_{0} G \Delta f, \bar{\alpha}^{2}=\frac{4 k T_{0} R_{0} \Delta f}{1+\omega^{2} C_{0} R_{0}^{2}}$

Using (4) expressions for $P^{2}$ can be written

$P^{2}=\frac{4 k T_{0} \Delta f\left(A_{0} G+G^{2}+\omega^{2} C^{2}\right)}{A_{0} \omega^{2} R_{0} \gamma^{2} \varepsilon_{1}^{2}}$

If we take into account that $\omega^{2} C^{2}<<G^{2}$ then we shall obtain:

$$
P^{2}=\frac{4 k T_{0} \Delta f\left(A_{0} T_{0}+C^{2}\right)}{A_{0} R_{0} \gamma^{2} \varepsilon_{1}^{2}}
$$

For a lithium niobate crystal the first term in this expression is much less than the second one, so we get

$$
P \approx \frac{2 C \sqrt{\frac{k T_{0} \Delta f}{R_{0}}}}{\sqrt{A_{0}} \varepsilon_{1} \gamma}
$$

where $C=C_{1} A_{0} d$

At $\varepsilon_{1}=0.48, R_{0}=10^{9} \mathrm{Ohm}, \gamma=2.4 \cdot 10^{-4} \mathrm{C} /\left(\mathrm{m}^{2} \mathrm{~K}\right), \Delta f=$ $=1 \mathrm{~Hz}, C_{1}=3.2 \cdot 10^{6} \mathrm{~J} /\left(\mathrm{m}^{3} \mathrm{~K}\right)$ we have $P \approx 1.3 \cdot 10^{-9} \mathrm{~W} / \mathrm{Hz}^{1 / 2}$.

The volt-watt sensitivity, calculated from the formula (4) at a value of input capacity of $C_{i n}=6 \cdot 10^{-12} \mathrm{~F}$ for a matched cascade on the field transistor switched under the scheme of the effluence repeater is $S=156 \mathrm{~V} / \mathrm{W}$. When an operational amplifier was used as a matched cascade switched under the scheme of a current-voltage converter, the detectivity of pyroelectric detector increases approximately twice and the wolt-watt sensitivity $S$ is enlarged $K_{n}$ times. $K_{n}=\omega R_{1} C_{c r}=20$, so $S \approx 3120 \mathrm{~V} / \mathrm{W}$. Therefore, the operational amplifier K544UD1A was selected as a matched cascade. It was switched under the scheme of current-woltage converter at the resistance $R=1 \mathrm{GOhm}$.

An experimental device was assembled consisting of an ethalon source: an absolutely black body (ABB) with a temperature of $300 \mathrm{~K}$, modulator with a modulation frequency of $20 \mathrm{~Hz}$, pyroelectric detector, which was under study, and selective potential galvanometer B6-9 for determining threshold and volt-watt sensitivity. Using the latter, the effective voltage of noises in a narrow frequency band at the input of a matched cascade of a pyroelectric detector was measured.
The volt-watt sensitivity was determined according to the ratio

$S=U_{c} /\left(A_{0} \Phi_{0}\right)$

and the threshold sensitivity $P$ according to the ratio

$P=U_{N} /\left[S\left(\Delta f_{0.5}\right)^{1 / 2}\right]$

where $\Delta f_{0.5}$ is the passband of a selective potential galvanometer at a level 0.5 from value of a signal at frequency $20 \mathrm{~Hz}, \Phi_{0}$ the density of a black-body radiation power at a distance of $20 \mathrm{~cm}$ from an outlet hole with a diameter of $10 \mathrm{~mm}, U_{N}$ the effective noise voltage, which was measured using an integrating sphere with a large time constant.

As results of the measurements, we have determined main characteristics of two examples of the pyroelectric detector model on the basis of lithium niobate (№1) and lithium tantalate crystals (№2) as compared to BMC-3 $\mathrm{Ni}$ bolometer. The results are summarized in Table 1 . So, the characteristics of designed pyroelectric detectors considerably exceed those for nickel bolometers, which are widely used in IR spectrophotometers to register radiation.

\section{Spectral sensitivity of the recording systems on the basis of designed pyroelectric detectors}

The spectral sensitivity $S_{1}=\Delta R / \Delta P_{1}$ of designed pyroelectric detectors was determined using a recording system based on a IKS-12 spectrophotometer. The known methods of measurement are conventionally divided into two groups. The first group comprises methods in which the detector under study should be calibrated using a radiation source with known distribution of radiation power coming from a monochromator with the predetermined transmission function. The second group are the methods in which spectral sensitivities of a detector under study and reference one are compared. As a reference detectors, a radiation calorimeters or cavernous detectors can be used.

The application of the first method is considerably difficult, because determining the power distribution of a radiation source and transmission function for any monochromator is quite a complicated problem.

The difficulties of the second method consist in selection of the reference detector. This problem was solved as follows. A number of non-selective detectors were investigated. Among them there were pyroelectric detectors with a mirror integrating hemisphere, with a flat receiving surface, conical, wedge and as a complete sphere. Then their relative spectral sensitivities were measured. It was obtained that the spectral sensitivity of such detectors are nonselective in the area $\lambda=1-15 \mu \mathrm{m}$. Among them the detector as a mirror hemisphere with a flat sensing element coated by golden niello was selected as a reference. It is necessary to note that theoretical calculations show that the pyroelectric detectors with a mirror integrating hemisphere in the region of $\lambda=1.5-15 \mu \mathrm{m}$ is 
V.O. Lysyuk et al.: Investigations of characteristics of metal film...

Table 1

\begin{tabular}{lllllll}
\hline \hline № & Parameter & Symbol & Dimension & Detector № 1 & Detector№ 2 & Bolometer BMC-3 \\
\hline 1 & Capacity of sensing element & $C$ & $\mathrm{pF}$ & 162 & 127 & - \\
\hline 2 & Size of the accepting zone & $A_{0}$ & $\mathrm{~mm}^{2}$ & 8 & 8 & 0.76 \\
\hline 3 & Output signal & $U_{S}$ & $\mathrm{~V}$ & $27 \cdot 10^{-3}$ & $26 \cdot 10^{-3}$ & - \\
\hline 4 & Effective noise voltage & $U_{N}$ & $\mathrm{~V}$ & $1.5 \cdot 10^{-6}$ & $3 \cdot 10^{-6}$ & - \\
\hline 5 & Volt-watt sensitivity & $S$ & $\mathrm{~V} / \mathrm{W}$ & $2.7 \cdot 10^{3}$ & $2.6 \cdot 103$ & 11 at $R_{1}=200 \mathrm{O} \mathrm{hm}$ \\
\hline 6 & Threshold sensitivity & $P$ & $\mathrm{~W} / \mathrm{Hz}^{1 / 2}$ & $5.5 \cdot 10^{-9}$ & $1.1 \cdot 10^{-9}$ & $1.0^{-9} \cdot 10^{-9}$ \\
\hline 7 & Time constant & $t$ & $\mu \mathrm{s}$ & 5 & 12 & $22 \cdot 10^{3}$ \\
\hline 8 & Maximum flux of radiation & $\mathrm{W}_{\max }$ & $\mathrm{W} / \mathrm{m}^{2}$ & $5 \cdot 10^{7}$ & $3 \cdot 10^{7}$ & - \\
\hline \hline
\end{tabular}

nonselective owing to large absorption of the surface of the detector and the use of a mirror hemisphere. Using this detector as a reference, it is possible to measure relative spectral sensitivity $S(\lambda)$ of a pyroelectric detector of any other design. Then, if necessary, it is possible to measure an absolute spectral sensitivity $S_{0}\left(\lambda_{0}\right)$ for a fixed wavelength $\lambda_{0}$ (as a rule, that of laser radiation). Then the curve of absolute spectral sensitivity $S_{\text {abs }}(\lambda)$ of the detector under study can be determined by the relation

$S_{\mathrm{abs}}(\lambda)=S_{0}\left(\lambda_{0}\right) \frac{S(\lambda)}{S_{\max }\left(\lambda^{\prime}\right)}$

where $S_{\max }\left(\lambda^{\prime}\right)$ is the peak of relative spectral sensitivity which is referred to a wavelength $\lambda$.

We have investigated spectral sensitivity of known detectors: conical, flat, transversal and longitudinal, coated by golden niello and by nickel-chromium film with a thickness of $40 \mathrm{~nm}$, as well as by nickel with the same thickness, implanted by $\mathrm{Ar}^{+}$ions with energies of $100 \mathrm{keV}$. The values of integral sensitivity of the detectors, studied with the count of a modulation factor $\beta=0.448$ are listed in Table 2.

The spectral sensitivity $S(\lambda)$ of the studied detector was determined as the relation of electrical signals of the studied $I_{\text {stud }}(\lambda)$ and the reference detector $I_{\text {ref }}(\lambda)$, namely $S(\lambda)=I_{\text {stud }}(\lambda) / I_{\text {ref }}(\lambda)$ (the curves $S(\lambda)$ are normalized).

The measured curves describing spectral sensitivities of different type pyroelectric detectors compared to that for BMC-3 Ni bolometer are shown in Fig.1. It is seen that the spectral sensitivity of hemisphere, conical, and also coated with golden niello detectors do not depend on the wavelength within a broad spectral range $\lambda=0.2$ $15 \mu \mathrm{m}$. The detectors coated by $\mathrm{Ni}-\mathrm{Cr}$ absorptive film are selective in this spectral range. And the detectors coated by ion implanted Ni film have a weaker selectivity, particularly, in the short-wave range and medium IR region $\lambda=2-9 \mu \mathrm{m}$ they are not selective. It is necessary to note that the BMC-3 bolometer has also weak selectivity.

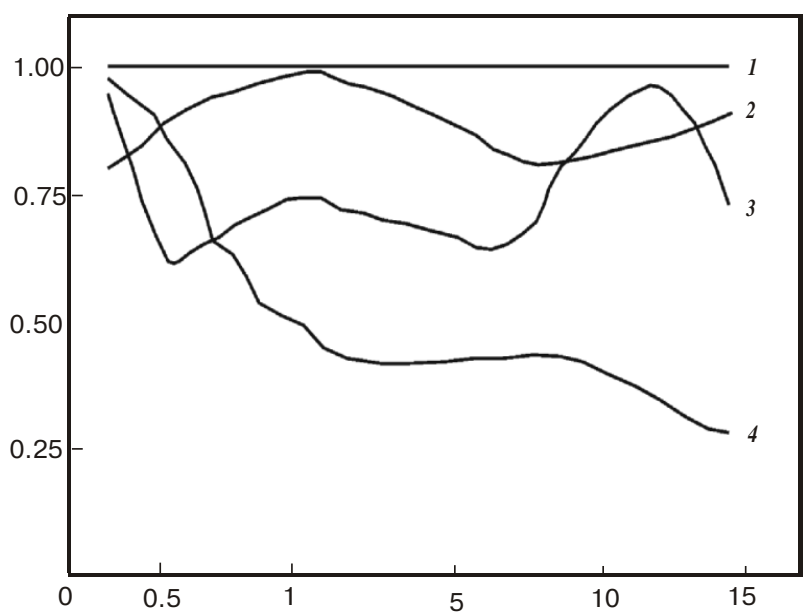

Fig. 1. Spectral sensitivity of pyroelectric detectors coated by the golden niello absorbing film (I), Ni-Cr absorbing film (3), $\mathrm{Ni}$ absorbing film implanted by $\mathrm{Ar}^{+}$ions (4) in comparison with that for serial bolometer BMC-3 (2).

Table 2

\begin{tabular}{|c|c|c|c|c|c|c|}
\hline Type of pyroelectric detector & Cavernous, & $\begin{array}{l}\text { Conical } \\
\text { hemispherical }\end{array}$ & $\begin{array}{l}\text { Flat, } \\
\text { transversal, } \\
\text { coated by } \\
\text { golden niello }\end{array}$ & $\begin{array}{l}\text { Flat, } \\
\text { longitudinal, } \\
\text { coated by } \\
\text { golden niello }\end{array}$ & $\begin{array}{l}\text { Flat, } \\
\text { longitudinal, } \\
\text { coated by } \\
\text { Ni-Cr film } \\
\end{array}$ & $\begin{array}{l}\text { Flat, longitudinal, } \\
\text { coated by Ni film, } \\
\text { implanted by } \\
\text { Ar+ ions }\end{array}$ \\
\hline No. & 1 & 2 & 3 & 4 & 5 & 7 \\
\hline$S_{0}, \times 10^{3} \mathrm{~V} / \mathrm{W}(f=20 \mathrm{~Hz})$ & 2.40 & 1.00 & 1.32 & 3.00 & 2.30 & 3.50 \\
\hline
\end{tabular}




\section{V.O. Lysyuk et al.: Investigations of characteristics of metal film...}

\section{Conclusions}

The characteristics and parameters (time constant, spectral sensitivity, threshold sensitivity, effective noise voltage, radiation stability) of pyroelectric detectors fabricated of various materials are measured and determined. The advantages of the detector with Ni absorptive film implanted by $\mathrm{Ar}^{+}$ions are exhibited. These have large radiation stability, high sensitivity and small lag. A comparison of the characteristics of such detector and those of BMC-3 bolometer, which is used in the majority of IR spectrophotometers, has shown a considerable advantage of the pyroelectric, including weak selectivity in shortwave range and medium IR region $(\lambda=2-9 \mathrm{~mm})$. These advantages make the use of the pyroelectric detectors with a $\mathrm{Ni}$ absorptive film implanted by $\mathrm{Ar}^{+}$ions in recording systems of IR engineering to be promising.

\section{References}

1. L.S. Kremenchugsky, O.V. Roytsyna, Pyroelectric detectors, Kyiv (1982), p. 1.

2. L.S. Kremenchugsky, O.V. Roytsyna, System of parameters and characteristics of thermal photodetectors, Kyiv (1983), p. 1 .

3. O.V. Vakulenko, L.S. Golovinskaya, O.V. Elfimov, at all, JPS 51, №1, (1989), p. 140.

4. O.V. Vakulenko, O.V. Elfimov, L.S. Kremenchugskiy, at all, JPS, 53, №1, (1990), p. 159.

5. L.V. Poperenko, T. Lohner, V.S. Staschuk, V.O. Lysyuk, Metalofiz. Noveyshie Technol., V.23, (2001), p. 199.

6. M. Fried, T. Lohner and P. Petric, Handbook of Surfaces and Interfaces of Materials, Academic Press, San Diego (2001), v.4, p. 335 .

7. J.S. Willams. Ion implantation and beam technology, Naukova dumka, Kyiv (1988), p. 1. 